\title{
Three dimensional ultrastructure of young osteocyte and osteocyte lacuna
}

\author{
Kazuyuki Segawa, Shozo Kitamura, Sigenori Taniuchi \\ and Reiji Takiguchi \\ The First Department of Oral Anatomy, School of Dentistry, Showa University, \\ 1-5-8 Hatanodai, Shinagawa-ku, Tokyo 142, Japan
}

[Accepted for publication: March 19, 1985]

Key words: organelle/young osteocyte/osteocyte lacuna/dilute osmium maceration/three dimensional ultrastructure

Osteocytes have been generally interpreted as cells in which osteoblasts with abundant organelles in relation to collagen synthesis were buried within the unmineralized bone matrix formed by osteoblasts themselves during the stage of bone matrix formation. Dudley et al. ${ }^{1)}$ named the osteocyte immediately after impaction within the bone matrix as "osteoid osteocyte" and found their resemblances with formative osteoblast organelles. Some of the isolated osteocytes accompanying bone mineralization show a little decrease in the amount of organelles thereby, which were named "young osteocyte" by Baud ${ }^{2)}$, and Jande ${ }^{3)}$ described them as "formative osteocyte" because unmineralized fibrillar structures were contained within pericellular zone of osteocyte lacuna in spite of mineralization of matrix around their lacuna. For the purpose of demonstrating such osteocytes and pericellular matrix on three dimensional views, by means of our modified method ${ }^{8,9)}$ based on the osmiumdimethyl sulfoxide--osmium method (the ODO method) devised by Tanaka et al. ${ }^{10)}$, we examined in detail with a high resolution scanning electron microscope.

\section{Materials and Methods}

As materials, the mandibular bodies of 20 newborn Wistar rats (7 days old) weighing $15-20 \mathrm{~g}$ were used. These rats were anesthetized with intraperitoneal injection of sodium pentobarbital and fixed by trans- cardiac perfusion with $2.5 \%$ glutaraldehyde buffered 1/15 $\mathrm{M}$ phosphate buffer ( $\mathrm{pH} 7.4$ ). After perfusion, the mandibles were dissected, cut into pieces, and placed in a mixture of $2.5 \%$ glutaraldehyde and $12.5 \%$ dimethyl sulfoxide (DMSO) in the same buffer overnight at $4^{\circ} \mathrm{C}$. The tissues were then freezecracked with congealed 50\% DMSO under liquid-nitrogen temperature. The cytoplasmic matrices of cracked cells were macerated and removed in buffered $0.1 \%$ osmium tetroxide for $72 \mathrm{~h}$ at $20^{\circ} \mathrm{C}$ to expose the membranous cell organelles, and the osteocytes and the amorphous organic matrices of the cracked surfaces were digested with Sigma type I. trypsin $0.5 \mathrm{mg} / \mathrm{ml}$, type V. hyaluronidase $1 \mathrm{mg} / \mathrm{ml}$ to expose the osteocyte lacunar walls. Next the specimens were conductive-stained with $1 \%$ osmium tetroxide and $2 \%$ tannic acid filtered using $0.2 \mu \mathrm{m}$ mili-pore, followed by dehydration by graded ethanols, critical point drying and ion sputter coating with platinum coating up to thickness $40 \AA$ by a polaron E-5450, then examined with a field emission type scanning electron microscope (Hitachi S-700).

\section{Results and Discussion}

Within the unmineralized osteoid layer close to the osteoblast layer, in which osteoid osteocytes for most part of enclosed with osteoid existed, and within the mineralized bone layer of the deep layer, the so-called young and formative osteocytes ${ }^{1-5)}$ were 
packed up. In the bones of young rats, these osteocytes seemed to be found numerously because many of osteocytes which appeared in the rat bone at this stage possessed considerably developed organelles. Most of the young osteocytes within the mineralized bone matrix were smaller than formative osteoblasts, therefore, had less components but of same position and similar to in formative osteoblasts $^{1-7)}$. In the juxtanuclear Golgi areas, Golgi apparatuses were not so welldeveloped in osteocytes as in osteoblasts, and consisted of stacks of several flattened, extensive cisterns, dilated vacuoles, many scattered vesicles. Some mitochondria which showed round profile of $0.5-0.7 \mu \mathrm{m}$ in diameter were dispersed throughout the cytoplasm. Rough cndoplasmic reticulum were well-developed in the areas adjacent to Golgi region, particularly, in the areas opposite direction to eccentrically located nucleus (Fig. 1, 2). Golgi apparatus consisted of stacks of $3-4$ cisterns with flattened or dilated lumen (Fig. 3). Rough endoplasmic reticulum were located as in osteocytes as in osteoblasts, and showed stacks of cisterns associated with ribosome as osteoblasts. However, they tended to decrease in amount (Fig. 4). In the border zone between the osteocyte and the osteocyte lacunar wall, the spaces containing unmineralized collagen fibrils were shown conspicuously. In the inner areas of plasma membrane, rough endoplasmic reticulum were assembled (Fig. 5). The matrix of lacunar wall consisting of unmineralized collagen fibrils was obvious, by means of digestion of the osteocytes and the amorphous organic matrices with the enzyme after freeze-cracking (Fig. 6). The collagen fibrils formed in these exposed surfaces of lacunar wall, coincided with the collagen fibrils contained within the zone of the spaces around the osteocytes described above Fig. 5. With regard to contents of the pericellular zone of osteocyte, many findings have been revealed $^{1-6,11-13)}$. Particularly at the stage of young and formative osteocyte, in spite of mineralization of perilacunar areas, intralacunar and pericellular zone contained collagen fibrils ${ }^{3,4,11-13}$, therefore, such collagen fibrils have been assumed to derive from the osteocyte possessed a faculty of collagen synthesis. The collagen fibrils constituting the unmineralized surface of osteocyte lacunar wall were arranged either irregular or regular in appearance. They have been assumed as apt to change gradually from irregular into regular arrangement ${ }^{12,13)}$.

As for the present, the method of both removal of cytoplasmic matrices by a dilute osmium maceration and digestion of organic matrices except for fibrillar matrices by an enzyme such as trypsin, hyaluronidase, may be applied to investigate the correlation with the osteocyte organelles and the structural features of their lacunar walls on scanning electron microscope.

\section{References}

1) Dudley, H. R. and Spiro, D.: The fine structure of bone cells. J. Biophys. Biochem. Cytol. 11: 627-649, 1961.

2) Baud, C. A.: Submicroscopic structure and functional aspects of the osteocyte. Clin. Orthop. Related Res. 56: 227-236, 1968.

3 ) Jande, S. S.: Fine structural study of osteocyte and their surrounding bone matrix with respect to their age in young chicks. J. Ultrastruct. Res. 37: 279-300, 1971.

4) Jande, S. S. and Bélanger, L. F.: Electron microscopy of osteocytes and the pericellular matrix in rat trabecular bones. Calcif. Tiss. Res. 6: 280-289, 1971.

5) Luk, S. C., Napajaroonsri, C. and Simon, G. T.: The ultrastructure of endosteum: A topographic study in young adult rabbits. J. Ultrastruct. Res. 46: 165-183, 1974.

6) Cameron, D. A.: The ultrastructure of bone. In: The Biochemistry and Physiology of bone (G. H. Bourne, ed.) 2nd eds., p. 191-236, Academic Press, New York, 1972.

7) Cameron, D. A.: The Golgi apparatus in bone and cartilage cells. Clin. Orthop. Related Res. 58: 191-211, 1968.

8) Segawa, K., Taniuchi, S. and Takiguchi, R.: Three dimensional ultrastructure of formative osteoblast organelles. Jpn. J. Oral Biol. 27: 334-337, 1985.

9) Segawa, K., Debari, K., Sasaki, T. and Takiguchi, R.: High-resolution scanning electron microscopy of the cells of mineralized tissues. J. Electron Microsc. Technique. 2: 271-272, 1985.

10) Tanaka, K. and Naguro, T.: High resolution 
scanning electron microscopy of cell organelles by a new specimen preparation method. Biomed. Res. 2 (Suppl.) : 63-70, 1981.

11) Wassermann, F. and Yaeger, J. A.: Fine structure of the osteocyte capsule and of the wall of the lacunae in bone. Z. Zellforsch. 67: 636-652, 1965.

12) Egawa, K., Suzuki, I. and Takiguchi, R.:
Fine structure of the osteocyte lacunar wall. Bull. Tokyo Dent. Coll. 21 : 127-135, 1980. 13) Segawa, K., Sugiyama, H. and Yokota, Y.: Studies on three dimensional ultrastructures of uncalcified layer of osteocyte lacunar walls (in Japanese). Jpn. J. Oral Biol. 26: 176$184,1984$.

\section{Explanation of figures}

Fig. 1 Young osteocyte possessed well-developed organelles in compact bone matrix (BM). Nucleus ( $\mathrm{N})$.

Fig. 2 Golgi apparatuses (G) consisting of several cisterns, vacuoles and vesicles in the area closest to nucleus $(\mathrm{N})$, their surrounding rough endoplasmic reticulum (RER), and mitochondria $(\mathbf{M})$ in dispersion throughout the cytoplasm.

Fig. 3 Golgi apparatus consisting of stacks of 3-4 cisterns with flattened and dilated lumen.

Fig. 4 Some cisterns of rough endoplasmic reticulum with structures resembling ribosomes.

Fig. 5 The border zone between osteocyte and their surrounding bone matrix (BM). Spaces between the plasma membrane of osteocyte and the edge of lacunar wall contain collagn fibrils (CO). Rough endoplasmic reticulum (RER) assembled in the inner area of plasma membrane.

Fig. 6 Lacunar wall surface which the osteocyte was digested by the enzyme. The matrix of lacunar wall is constituted with collagen fibrils appeared irregular and reticular arrangement. 

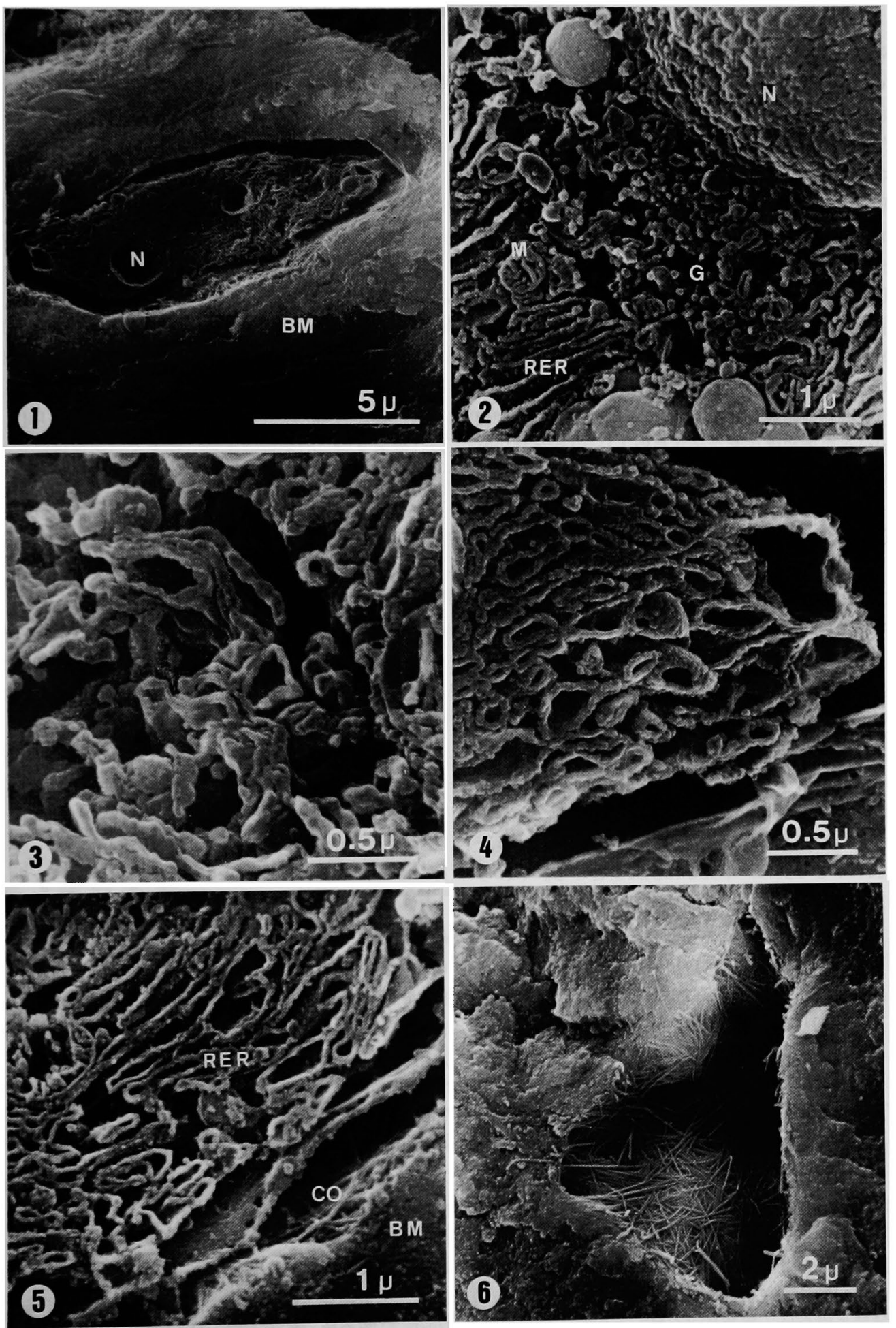